\title{
Article \\ Factors Associated with Daptomycin-Induced Eosinophilic Pneumonia
}

\author{
Kazuhiro Ishikawa ${ }^{1, *}$, Takahiro Matsuo ${ }^{1}$, Yasumasa Tsuda ${ }^{2}$, Mahbubur Rahman ${ }^{3}$, Yuki Uehara ${ }^{1,4,5,6}$ (D) \\ and Nobuyoshi Mori ${ }^{1}$
}

Citation: Ishikawa, K.; Matsuo, T.; Tsuda, Y.; Rahman, M.; Uehara, Y.; Mori, N. Factors Associated with Daptomycin-Induced Eosinophilic Pneumonia. Antibiotics 2022, 11, 254. https://doi.org/10.3390/ antibiotics11020254

Academic Editors: Marc Maresca and Francois Vandenesch

Received: 18 December 2021

Accepted: 10 February 2022

Published: 16 February 2022

Publisher's Note: MDPI stays neutral with regard to jurisdictional claims in published maps and institutional affiliations.

Copyright: (C) 2022 by the authors. Licensee MDPI, Basel, Switzerland. This article is an open access article distributed under the terms and conditions of the Creative Commons Attribution (CC BY) license (https:// creativecommons.org/licenses/by/ $4.0 /)$.
1 Department of Infectious Diseases, St. Luke's International Hospital, Tokyo 104-8560, Japan; takahirom1226@gmail.com (T.M.); yukiue@luke.ac.jp (Y.U.); morinob@luke.ac.jp (N.M.)

2 Department of Pharmacy, St. Luke's International Hospital, Chuo-ku, Tokyo 104-8560, Japan; tsuyasu@luke.ac.jp

3 Division of Epidemiology, Graduate School of Public Health, St. Luke's International University, Tokyo 104-0045, Japan; rahman@luke.ac.jp

4 Department of Clinical Laboratory, St. Luke's International Hospital, Tokyo 104-8560, Japan

5 Department of Microbiology, Juntendo University Faculty of Medicine, Tokyo 113-8421, Japan

6 Department of General Medicine, Juntendo University Faculty of Medicine, Tokyo 113-8421, Japan

* Correspondence: ishikawakazuhiro@gmail.com; Tel.: +81-3-3541-5151

\begin{abstract}
The risk factors for eosinophilic pneumonia (EP) remain unclear. We investigate the characteristics of patients with daptomycin (DAP)-induced EP and conducted a retrospective observational study. A total of 450 patients aged $\geq 18$ years who received DAP (25 DAP with EP, 425 DAP without $\mathrm{EP})$ were included. The median duration from the first DAP administration to EP onset was 18.0 days. Definite, probable, and possible DAP-induced EP were diagnosed in 0, 9, and 16 patients, respectively. The median age (DAP with EP, 72.0 years; DAP without EP, 64.0 years), DAP dosage/body weight (BW) $(9.00$ vs. $7.50 \mathrm{mg} / \mathrm{kg})$, blood eosinophil count (cells $/ \mu \mathrm{L})(419 \mathrm{vs}$. 96), and the percentage of hemodialyzed patients $(40.0 \%$ vs. $13.4 \%)$ were significantly higher in patients with EP than in patients without EP in the univariate analysis. In separate multivariate logistic regression analyses, age (odds ratio (OR), 1.03; 95\% confidence interval (CI), 1.00-1.05), DAP dosage/BW (OR, 1.61; 95\% CI, 1.25-2.07), and hemodialysis (OR, 4.42; 95\% CI, 1.86-10.5) were significantly associated with DAP-induced EP. Clinicians may need to consider the potential factors associated with EP, especially in older patients, patients on hemodialysis, or patients who receive $>9.00 \mathrm{mg} / \mathrm{kg}$ of DAP.
\end{abstract}

Keywords: daptomycin; eosinophilic pneumonia; Staphylococcus aureus; methicillin-resistant Staphylococcus aureus

\section{Introduction}

Methicillin-resistant Staphylococcus aureus (MRSA) infections have high mortality rates of $15-25 \%$ [1,2]. The standard treatment for MRSA bacteremia is vancomycin (VCM) or daptomycin (DAP) [3]. DAP is a cyclic lipopeptide antimicrobial agent used to treat skin and soft tissue infections and infective endocarditis. DAP is bactericidal and is considered to be more effective than VCM for MRSA infections; this was observed in a previous study where a higher the duration of negative blood cultures was observed with DAP than VCM [4]. DAP has a high protein-binding concentration of 90-93\%, a long half-life in blood (about $8.5 \mathrm{~h}$ ), and is excreted by the kidneys. For patients with a creatinine clearance $(\mathrm{CCr})$ of $\geq 30 \mathrm{~mL} / \mathrm{min}$, the DAP dosage is $4-10 \mathrm{mg} / \mathrm{kg}$ in a single daily dose or once every $48 \mathrm{~h}$ for patients with a $\mathrm{CCr}<30 \mathrm{~mL} / \mathrm{min}$ or patients on dialysis [5]. The elevation of creatine kinase (CK) levels and eosinophilic pneumonia (EP) are side effects of DAP [5].

EP are characterized by marked accumulations of infiltrating eosinophils in the alveolar space and the interstitium. Although EP can lead to hypoxemia and serious complications, very few studies have been published on its risk factors, including literature reviews 
and a study with a small sample size [6,7]. These studies reported that older age and a total DAP dose $>10 \mathrm{~g}$ are risk factors for DAP-induced EP. Conversely, it has been reported that patients with obesity are more likely to experience adverse effects from beta-lactam antimicrobials due to an increased volumetric distribution (Vd) [8]. However, whether obesity is a risk factor for DAP-induced EP is unknown. In this study, we retrospectively investigate the risk factors for DAP-induced EP.

\section{Results}

A total of 450 patients treated with DAP between June 2011 and July 2020 were selected. Of those, 25 developed EP after the first DAP administration (EP with DAP), while 425 did not (EP without DAP) (Figure 1).

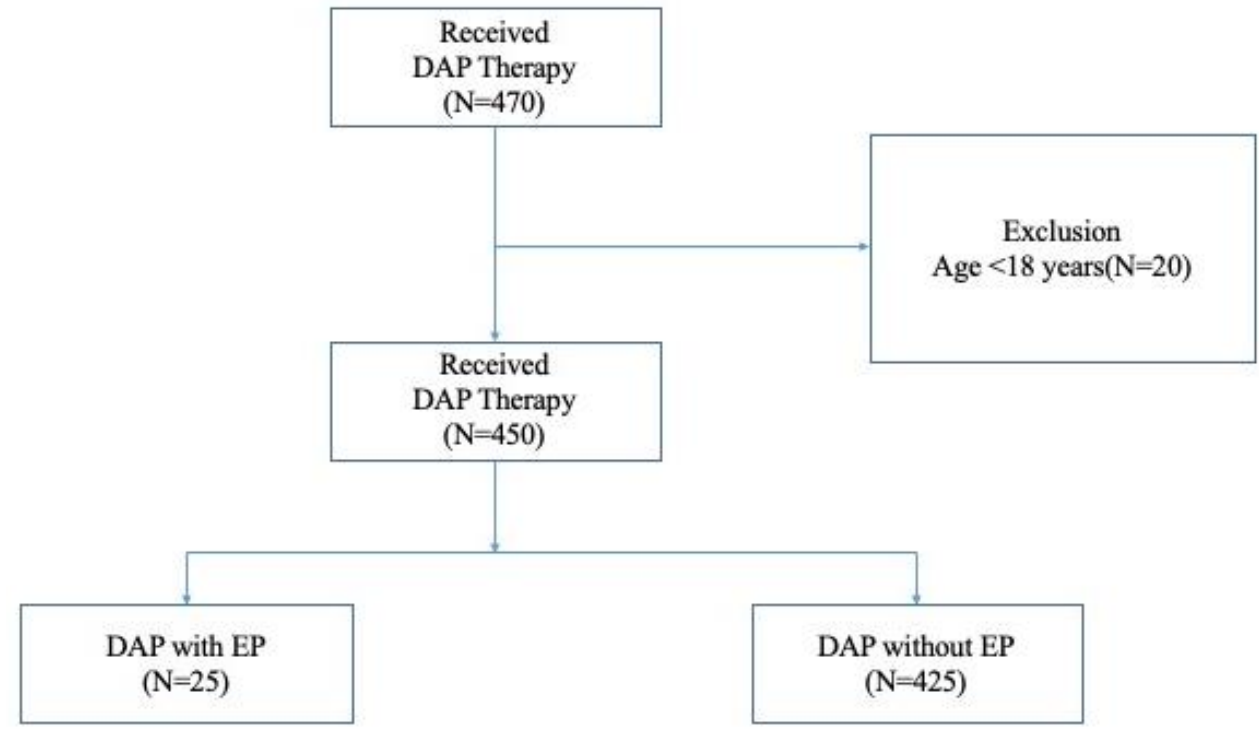

Figure 1. Patient selection flow chart. Abbreviations: DAP, daptomycin; EP, eosinophilic pneumonia.

The median duration from the first DAP dose administration to EP onset among the 25 patients with EP was 18.00 (interquartile range (IQR), 26.5; minimum-maximum, (3-49) days. The number of patients with definite, probable, and possible EP is as follows (Table 1).

Table 1. Characteristics of patients with DAP-induced EP.

\begin{tabular}{ll}
\hline Patient Characteristics & EP $(\boldsymbol{n}=\mathbf{2 5})$ \\
\hline $\begin{array}{l}\text { Median duration from first DAP } \\
\text { administration to EP onset (IQR, } \\
\text { minimum-maximum), days }\end{array}$ & $18.00(26.5,3-49)$ \\
\hline Definite, $n(\%)$ & $0(0 \%)$ \\
\hline Probable, $n(\%)$ & $9(36 \%)$ \\
\hline $\begin{array}{ll}\text { Possible, } n(\%) \text { Clinical improvement following } \\
\text { DAP withdrawal, } n(\%)\end{array}$ & $16(64 \%)$ \\
\hline Abbreviations: SD, standard deviation; DAP, daptomycin; EP, eosinophilic pneumonia, IQR: interquartile range.
\end{tabular}

No patients underwent BAL, so the number of patients with definite EP was zero. Fourteen patients with possible EP improved clinically after the discontinuation of DAP, but two patients died of heart failure.

Table 2 shows the results of the univariate analysis of the baseline and clinical characteristics of both groups. 
Table 2. Comparison of baseline and clinical characteristics between patients with DAP-induced EP and patients without DAP-induced EP.

\begin{tabular}{|c|c|c|c|}
\hline & DAP with EP $(n=25)$ & DAP without EP $(n=425)$ & $p$-Value \\
\hline Age in years, median (IQR) & $72.0(26)$ & $64.0(32)$ & 0.030 \\
\hline Male, $n(\%)$ & $18(72.0 \%)$ & $249(58.6 \%)$ & 0.185 \\
\hline $\mathrm{BW}(\mathrm{IQR}), \mathrm{kg}$ & $53.8(17.0)$ & $58.9(21.6)$ & 0.079 \\
\hline $\mathrm{BMI}(\mathrm{IQR}), \mathrm{kg} / \mathrm{m}^{2}$ & $20.0(6.3)$ & $21.9(6.0)$ & 0.040 \\
\hline $\mathrm{BW} / \mathrm{IBW}>1, n(\%)$ & $9(36.0)$ & $192(45.2)$ & 0.297 \\
\hline $\mathrm{BW} / \mathrm{ABW}>1, n(\%)$ & $11(44.0)$ & $240(56.5)$ & 0.203 \\
\hline Heart failure, $n(\%)$ & $10(40.0)$ & $134(31.5)$ & 0.378 \\
\hline Diabetes mellitus, $n(\%)$ & $6(24.0)$ & $147(34.6)$ & 0.277 \\
\hline Respiratory disease, $n(\%)$ & $18(72.0)$ & $255(60.0)$ & 0.233 \\
\hline Myocardial infarction, $n(\%)$ & $3(12.0)$ & $48(11.3)$ & 0.914 \\
\hline Collagen disease, $n(\%)$ & $2(8.00)$ & $41(9.6)$ & 0.785 \\
\hline Hepatic disease, $n(\%)$ & $5(20.0)$ & $68(16.0)$ & 0.598 \\
\hline Solid tumor, $n(\%)$ & $11(44.0)$ & $157(36.9)$ & 0.478 \\
\hline Hematological malignancy, $n(\%)$ & $1(4.00)$ & $58(13.6)$ & 0.165 \\
\hline Chronic kidney disease, $n(\%)$ & $9(36.0)$ & $89(20.9)$ & 0.076 \\
\hline Hemodialysis, $n(\%)$ & $10(40.0)$ & $57(13.4)$ & $<0.001$ \\
\hline Cerebrovascular disease, $n(\%)$ & $2(8.00)$ & $66(15.5)$ & 0.307 \\
\hline Hypertension, $n(\%)$ & $16(64.0)$ & $210(49.4)$ & 0.156 \\
\hline $\mathrm{HIV}, n(\%)$ & $0(0)$ & $1(0.2)$ & 0.808 \\
\hline Immunosuppressor, $n(\%)$ & $1(4.0)$ & $35(8.2)$ & 0.448 \\
\hline Biological agent, $n(\%)$ & $0(0)$ & $1(0)$ & 0.808 \\
\hline Steroid, $n(\%)$ & $5(20.0)$ & $98(23.1)$ & 0.723 \\
\hline Statin, $n(\%)$ & $6(24.0)$ & $86(20.2)$ & 0.650 \\
\hline Shock (SBP < 90), $n(\%)$ & $7(28.0)$ & $158(37.2)$ & 0.355 \\
\hline qSOFA > $2, n(\%)$ & $11(44.0)$ & $193(45.4)$ & 0.441 \\
\hline DAP dosage (IQR) (mg/day) & $525(235)$ & $350(230)$ & 0.046 \\
\hline DAP dosage /BW (IQR) (mg/kg) & $9.00(2.7)$ & $7.50(2.60)$ & $<0.001$ \\
\hline DAP dosage/IBW (IQR) (mg/kg) & $8.90(2.70)$ & $7.40(3.84)$ & 0.025 \\
\hline DAP dosage/ABW (IQR) (mg/kg) & $8.93(2.33)$ & $7.44(3.25)$ & 0.020 \\
\hline Total dosage of DAP (IQR) (mg) & $7875(9300)$ & $4800(8100)$ & 0.032 \\
\hline WBC (IQR) $(/ \mu \mathrm{L})$ & $8400(2950)$ & $7600(5600)$ & 0.625 \\
\hline Blood eosinophilia (IQR) $(/ \mu \mathrm{L})$ & $419(910)$ & $96(255)$ & $<0.001$ \\
\hline HGB (IQR) (g/dL) & $10.0(3.0)$ & $9.50(2.8)$ & 0.744 \\
\hline PLT (IQR) $(/ \mu \mathrm{L})$ & $21.2(15.8)$ & $21.1(20.0)$ & 0.643 \\
\hline BUN (IQR) (mg/dL) & $20.0(30.0)$ & $18.4(24.7)$ & 0.968 \\
\hline $\mathrm{sCr}(\mathrm{IQR})(\mathrm{mg} / \mathrm{dL})$ & $1.01(1.92)$ & $0.92(1.18)$ & 0.929 \\
\hline eGFR (IQR) (mL/min/1.73 m²) & $62.1(78.0)$ & $58.8(61.9)$ & 0.671 \\
\hline $\mathrm{CCr}(\mathrm{IQR})\left(\mathrm{mL} / \mathrm{min} / 1.73 \mathrm{~m}^{2}\right)$ & $68.2(66.1)$ & $58.9(82.4)$ & 0.361 \\
\hline LDH (IQR) (IU/1) & $231.5(155)$ & $227(139)$ & 0.450 \\
\hline CK (IQR) (IU.L) & $26(90)$ & $47(84)$ & 0.102 \\
\hline CRP (IQR) mg/dL & $10.6(15.8)$ & $5.39(9.2)$ & 0.010 \\
\hline
\end{tabular}


Table 2. Cont.

\begin{tabular}{|c|c|c|c|}
\hline & DAP with EP $(n=25)$ & DAP without EP $(n=425)$ & $p$-Value \\
\hline $\begin{array}{l}\text { Positive blood culture within one } \\
\text { month, } n(\%)\end{array}$ & $8(32.0)$ & $145(34.1)$ & 0.828 \\
\hline MRSA infection, $n(\%)$ & $4(16.0)$ & $29(6.8)$ & 0.087 \\
\hline CNS infection, $n(\%)$ & $2(8.0)$ & $58(13.6)$ & 0.420 \\
\hline Mortality at discharge, $n(\%)$ & $5(20.0)$ & $87(20.6)$ & 0.946 \\
\hline $\begin{array}{l}\text { Mortality within } 30 \text { days from DAP } \\
\text { administration, } n(\%)\end{array}$ & $4(16.0)$ & $59(13.9)$ & \\
\hline $\begin{array}{l}\text { Mortality within } 90 \text { days from DAP } \\
\text { administration, } n(\%)\end{array}$ & $4(16.0)$ & $76(17.9)$ & \\
\hline $\begin{array}{l}\text { ICU admission within } 30 \text { days from } \\
\text { admission, } n(\%)\end{array}$ & $5(20.0)$ & $75(17.6)$ & \\
\hline Mechanical intubation, $n(\%)$ & $5(20.0)$ & $65(15.3)$ & \\
\hline \multicolumn{4}{|c|}{$\begin{array}{l}\text { MRSA or CNS infection was defined as positive culture of blood, urine, abscess, soft tissue, etc. Abbreviations } \\
\text { DAP, daptomycin; EP, eosinophilic pneumonia; SD, standard deviation; BW, body weight; BMI, body mass } \\
\text { index; IBW, ideal body weight; ABW, adjusted body weight; systolic blood pressure; qsofa, quick Sequential } \\
\text { Organ Failure Assessment score; ICU, intensive care unit; HGB, hemoglobin; PLT, platelet; BUN, blood urea } \\
\text { nitrogen; sCr, serum creatinine; eGFR, estimated glomerular filtration rate; CCr, creatinine clearance; LDH, lactate } \\
\text { dehydrogenase; CK, creatine kinase; CRP, C-reactive protein; MRSA, methicillin-resistant Staphylococcus aureus } \\
\text { CNS, coagulase-negative Staphylococcus. }\end{array}$} \\
\hline
\end{tabular}

Compared to the DAP without EP group, the DAP with EP group had the following characteristics, which were statistically significant: a higher median age (IQR) (72.0 (26) vs. 64.0 (32) years, $p=0.030$ ), lower median body mass index (BMI) (IQR) (20.0 (6.3) vs. $\left.21.9(6.0) \mathrm{kg} / \mathrm{m}^{2}, p=0.040\right)$, greater proportion of hemodialyzed patients $(10(40 \%)$ vs. 57 $(13.4 \%), p<0.001)$, higher DAP dosage (IQR) (525 (235) vs. 350 (230) $\mathrm{mg} /$ day, $p=0.046)$, higher DAP dosage/body weight (IQR) (9.00 (2.7) vs. $7.50(2.60) \mathrm{mg} / \mathrm{kg}, p<0.001)$, higher dosage/ideal body weight (IBW) (IQR) (8.90 (2.70) vs. 7.40 (3.84) $\mathrm{mg} / \mathrm{kg}, p=0.025)$, higher dosage/adjusted body weight (ABW) (IQR) (8.93 (2.33) vs. $7.44(3.25) \mathrm{mg} / \mathrm{kg}, p=0.020)$, higher total dosage of DAP (IQR) (7875 (9300) vs. $4800(8100) \mathrm{mg}, p=0.032)$, higher total dosage of DAP (IQR)(7875 (9300) vs. 4800 (8100) $\mathrm{mg}, p=0.032)$, higher blood eosinophil count (IQR) $(419$ (910) vs. $96(255) / \mu \mathrm{L}, p<0.001)$, and higher C-reactive protein level (IQR) (10.6 (15.8) vs. $5.39(9.2) \mathrm{mg} / \mathrm{dL}, p=0.010)$. The DAP with EP group had a lower MRSA infection (defined as positive culture of blood, urine, abscess, soft tissue, and so on) proportion, although it was not statistically significant (4 (16.0\%) vs. $29(6.8 \%), p=0.087)$. The distribution of DAP dosage/BW $(\mathrm{mg} / \mathrm{kg}$ ) in DAP with EP is shown in Figure 2.

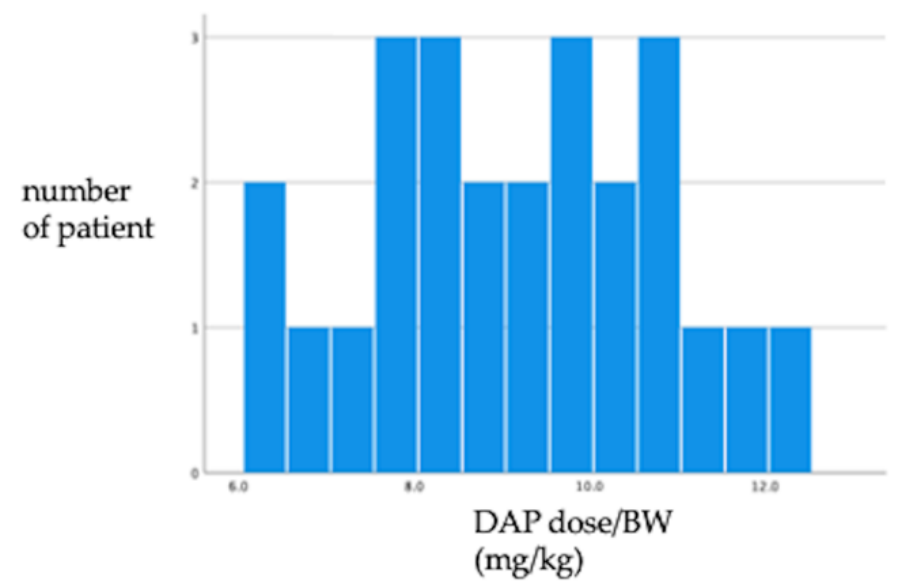

Figure 2. The distribution of DAP dosage / BW $(\mathrm{mg} / \mathrm{kg})$ in DAP with EP. Abbreviations: DAP, daptomycin; EP, eosinophilic pneumonia; BW, body weight. 
Separate multivariate logistic regression analyses were conducted for each of the characteristics after adjusting for age and BMI (Table 3). Age (odds ratio (OR), 1.03; 95\% confidence interval (CI), 1.00-1.05), DAP dosage/BW (OR, 1.61; 95\% CI, 1.25-2.07), and hemodialysis (OR, 4.42; 95\% CI, 1.86-10.5) were significantly associated with DAPinduced EP.

Table 3. Factors associated with DAP-induced EP based on the multivariable logistic regression analysis.

\begin{tabular}{ccc}
\hline & OR $(\mathbf{9 5} \% \mathrm{CI})$ & $p$-Value \\
\hline Age & $1.03(1.00-1.05)$ & 0.042 \\
\hline DAP dosage/BW $(\mathrm{mg} / \mathrm{kg})$ & $1.61(1.25-2.07)$ & $<0.001$ \\
\hline Hemodialysis & $4.42(1.86-10.5)$ & 0.010 \\
\hline MRSA infection & $2.04(0.63-6.62)$ & 0.235 \\
\hline
\end{tabular}

Abbreviations: DAP, daptomycin; EP, eosinophilic pneumonia; BW, body weight; BMI; ABW, adjusted body weight; MRSA, methicillin-resistant Staphylococcus aureus; OR, Odds Ratio; CI, Confidence Interval.

\section{Discussion}

In this study, we observed that hemodialysis and dosage/BW are associated with EP. The eosinophil count in bronchoalveolar lavage (BAL) is a criterion for the definite diagnosis of EP [6]. However, BAL could not be performed clinically due to the risk of worsening disease progression. Previously published studies used the blood eosinophil count as a criterion for possible EP [6]. In our study, the eosinophil count in blood was significantly higher in the DAP with EP group than in the DAP without EP group. Therefore, we assumed that the blood eosinophil count could be substituted for that of BAL for the diagnosis of EP.

The U.S. FDA identified six cases (patient age range, 60-87 years) of DAP-induced EP between 2004 and 2010 [9-12]. The patients developed EP 2-4 weeks after DAP initiation, while all seven patients reported improvement or resolution of symptoms after DAP discontinuation. Five of the seven patients were also treated with systemic corticosteroids, and two patients reported EP recurrence after the re-administration of DAP. In another retrospective study [7], the risk factors for DAP-induced EP were age $\geq 70$ years and duration of therapy $>14$ days. In our study, the median age and the median number of days from DAP initiation to EP onset were identified as risk factors. These results are consistent with those of a previously published report [13]. All the patients except two who died of heart failure improved clinically and rapidly after the treatment with DAP was discontinued. Therefore, none of the patients used steroids for the treatment of EP.

In our study, we found that hemodialysis, DAP dosage/BW, DAP dosage/IBW, DAP dosage/ABW, and total dosage of DAP are significant risk factors for EP. The reported dosage of DAP in a literature review was $4.4-8.0 \mathrm{mg} / \mathrm{kg} /$ day [6]. Nevertheless, EP was not observed among the participants of a prospective cohort study of patients with leftsided infective endocarditis treated with DAP at a dose of $9.2 \mathrm{mg} / \mathrm{kg} /$ day [4]. In an observational study including 102 patients with infectious endocarditis treated with a DAP dose of $8.2 \mathrm{mg} / \mathrm{kg}$, three patients developed EP [14]. In a retrospective study [7], a total dose of DAP > $10 \mathrm{~g}$ was observed as a risk factor in an unadjusted analysis. Hayes et al. hypothesized that DAP may cause EP by binding with the human pulmonary surfactant, resulting in its accumulation in the alveolar spaces at concentrations high enough to injure the epithelium and cause inflammation [10]. DAP has a very small volume of distribution of $7 \mathrm{~L}$, which is indicative of a very little tissue distribution [15]. According to the manufacturer's instructions, there is no need to adjust the DAP dosage for obese patients. However, when calculating the dosage/BW, it is recommended to use the IBW for hydrophilic drugs and the ABW for lipophilic drugs (mainly for obese people) [16]. In this study, we could not show the optimal dose of DAP for body types, such as obese and lean, but we observed that a high dose is a risk factor for EP. DAP clearance is performed by the kidneys. For patients on dialysis, it is slowly cleared from the body by hemodialysis 
(approximately 15\% of the administered dose is removed in over $4 \mathrm{~h}$ ) and peritoneal dialysis (approximately 11\% of the administered dose is removed in over $48 \mathrm{~h}$ ). The dosing interval for patients with a creatinine clearance of $<30 \mathrm{~mL} / \mathrm{min}$ should be decreased from every $24 \mathrm{~h}$ to every $48 \mathrm{~h}$. However, CK elevation may occur even in patients with renal dysfunction, despite appropriate dosages and intervals [17]. Therefore, EP should be anticipated when administering DAP to patients with renal dysfunction. If possible, other anti-MRSA antibiotics such as vancomycin should be used for hemodialyzed patients.

This study has several limitations. First, this study is based on a small number of events using a single-center observational study design. Therefore, prospective clinical studies are warranted to shed more light on the causal association between identified risk factors and DAP-induced EP. Second, although BAL is a mandatory criterion in the FDA definition of EP, all our patients did not undergo BAL. Since most of the patients had probable or possible EP, the possibility that EP may have had other etiologies than DAP use cannot be completely ruled out. However, blood eosinophil counts were significantly higher in the patients with DAP-induced EP. They improved clinically and rapidly after DAP discontinuation, suggesting that BAL may not be essential for diagnosis

\section{Materials and Methods}

\subsection{Study Design and Setting}

This was a single-center retrospective observational study conducted at St. Luke's International Hospital, a 520-bed teaching hospital in Tokyo.

\subsection{Inclusion and Exclusion Criteria}

Adult patients (aged $\geq 18$ years) who were hospitalized and treated with intravenous DAP between June 2011 and July 2020 were included. Eligible patients were identified from the hospital's electronic database. In this study, DAP-induced EP was classified as definite, probable, and possible, in accordance with the definition by Kim et al. [6] (Table 4)

Table 4. Criteria for definite, probable, and possible DAP-induced EP (6).

\section{Definite}

- Concurrent exposure to DAP

- Fever

- Dyspnea with an increased oxygen requirement or requiring mechanical ventilation

- New infiltrates observed on a chest X-ray or CT scan

- $\quad$ Bronchoalveolar lavage with $>25 \%$ eosinophils

- Clinical improvement following DAP withdrawal

\section{Probable}

- $\quad$ Concurrent exposure to DAP

- Dyspnea with increased oxygen requirement or requiring mechanical ventilation

- New infiltrates observed on a chest X-ray or CT scan

- Bronchoalveolar lavage $<25 \%$ eosinophils or peripheral eosinophilia *

- Clinical improvement following DAP withdrawal

* Peripheral eosinophilia was defined as a peripheral blood eosinophil level above the upper limit of the normal values of the reporting laboratory or a reported elevated blood eosinophil level without any record of the actual laboratory value.

\section{Possible}

- $\quad$ Concurrent exposure to DAP

- New infiltrates observed on a chest X-ray or CT scan

- $\quad$ Clinical improvement following DAP withdrawal or patient death

Abbreviations: DAP, daptomycin; CT, computed tomography.

The criteria for definite EP are similar to those of EP put forth by the United States Food and Drug Administration (U.S. FDA; all the criteria have to be met): concurrent exposure to DAP, fever, dyspnea with increased oxygen requirement or requiring mechanical 
ventilation, new infiltrates observed on a chest X-ray or computed tomography scan, BAL with $>25 \%$ eosinophils, and clinical improvement following DAP withdrawal.

\subsection{Data Collection}

Patient data were extracted from inpatient electronic medical records. The study variables included patient demographics (age and sex), comorbidities, use of antimicrobials other than DAP, DAP dosage, body weight, height, vital signs, laboratory test results, radiology imaging including chest radiography and computed tomography, microbiological findings, mortality, and complications including septic shock and mechanical intubation.

\subsection{Statistical Analyses}

Bivariate associations were assessed using $\chi^{2}$ and Fisher's exact test for categorical variables and the Mann-Whitney $U$ test for continuous variables. A significance level of 0.10 was set for variables in the univariate analysis to be included in the multivariate analyses. According to the results of the univariate analysis, separate multivariate logistic regression analyses were conducted for each of the characteristics after adjusting for age and BMI. All analyses were performed using SPSS 19.0 J statistical software (IBM Japan, Tokyo, Japan).

\subsection{Patient Consent Statement}

This study was approved by the Institutional Review Board of St. Luke's International Hospital in Tokyo, Japan (number: 20-R101). The requirement for patient consent was waived due to the study's retrospective nature.

\section{Conclusions}

Older patients, higher DAP dosage/BW, and hemodialysis were independent risk factors for DAP-induced EP.

Author Contributions: The manuscript was approved by all authors, and it is not under consideration elsewhere. All authors contributed to the work of this report. K.I. collected the clinical data and wrote the initial draft of the manuscript. Y.T., T.M., Y.U. and N.M. assisted in the study conception and design. K.I. and M.R. analyzed the data. M.R., Y.T., Y.U. and N.M. assisted in the interpretation of results and writing of the manuscript. All authors have read and agreed to the published version of the manuscript.

Funding: This research received no external funding.

Institutional Review Board Statement: This study was approved by the Institutional Review Board of St. Luke's International Hospital in Tokyo, Japan (number: 20-R101).

Informed Consent Statement: The requirement for patient consent was waived due to the study's retrospective nature. We complied with the Declaration of Helsinki, and the Ethical Guidelines for Medical Research Involving Human Subjects and gave consideration to the protection of human rights. Since this research is an observational study using existing samples and information, there was no direct intervention on the subject individuals, and the human rights of the individuals are protected.

Data Availability Statement: The data presented in this study are available on request from the corresponding author (K.I.) upon reasonable request.

Conflicts of Interest: The authors declare no conflict of interest.

\section{References}

1. Kourtis, A.P.; Hatfield, K.; Baggs, J.; Mu, Y.; See, I.; Epson, E.; Nadle, J.; Kainer, M.A.; Dumyati, G.; Petit, S.; et al. Vital Signs: Epidemiology and Recent Trends in Methicillin-Resistant and in Methicillin-Susceptible Staphylococcus aureus Bloodstream Infections-United States. MMWR Morb. Mortal. Wkly. Rep. 2019, 68, 214-219. [CrossRef] [PubMed]

2. Tong, S.Y.; Davis, J.S.; Eichenberger, E.; Holland, T.L.; Fowler, V.G. Staphylococcus aureus Infections: Epidemiology, Pathophysiology, Clinical Manifestations, and Management. Clin. Microbiol. Rev. 2015, 28, 603-661. [CrossRef] 
3. Liu, C.; Bayer, A.; Cosgrove, S.E.; Daum, R.S.; Fridkin, S.K.; Gorwitz, R.J.; Kaplan, S.L.; Karchmer, A.W.; Levine, D.P.; Murray, B.E.; et al. Clinical Practice Guidelines by the Infectious Diseases Society of America for the Treatment of Methicillin-Resistant Staphylococcus aureus Infections in Adults and Children. Clin. Infect. Dis. 2011, 52, e18-e55. [CrossRef] [PubMed]

4. Carugati, M.; Bayer, A.S.; Miró, J.M.; Park, L.P.; Guimarães, A.C.; Skoutelis, A.; Fortes, C.Q.; Durante-Mangoni, E.; Hannan, M.M.; Nacinovich, F.; et al. High-Dose Daptomycin Therapy for Left-Sided Infective Endocarditis: A Prospective Study From the International Collaboration on Endocarditis. Antimicrob. Agents Chemother. 2013, 57, 6213-6222. [CrossRef]

5. Highlights of Prescribing Information. These Highlights Do Not Include All the Information Needed to Use Cubicin Safely and Effectively. See Full Prescribing Information. for CUBICIN. Available online: https://www.merck.com/product/usa/pi_ circulars/c/cubicin/cubicin_pi.pdf (accessed on 6 December 2021).

6. Kim, p.W.; Sorbello, A.F.; Wassel, R.T.; Pham, T.M.; Tonning, J.M.; Nambiar, S. Eosinophilic Pneumonia in Patients Treated With Daptomycin: Review of the Literature and US FDA Adverse Event Reporting System Reports. Drug Saf. 2012, 35, 447-457. [CrossRef] [PubMed]

7. Soldevila-Boixader, L.; Villanueva, B.; Ulldemolins, M.; Benavent, E.; Padulles, A.; Ribera, A.; Borras, I.; Ariza, J.; Murillo, O. Risk Factors of Daptomycin-Induced Eosinophilic Pneumonia in a Population With Osteoarticular Infection. Antibiotics 2021, 10, 446. [CrossRef] [PubMed]

8. Hites, M.; Taccone, F.S.; Wolff, F.; Cotton, F.; Beumier, M.; De Backer, D.; Roisin, S.; Lorent, S.; Surin, R.; Seyler, L.; et al. CaseControl Study of Drug Monitoring of $\beta$-Lactams in Obese Critically Ill Patients. Antimicrob. Agents Chemother. 2013, 57, 708-715. [CrossRef] [PubMed]

9. Lal, Y.; Assimacopoulos, A.P. Two Cases of Daptomycin-Induced Eosinophilic Pneumonia and Chronic Pneumonitis. Clin. Infect. Dis. 2010, 50, 737-740. [CrossRef] [PubMed]

10. Hayes, D.; Anstead, M.I.; Kuhn, R.J. Eosinophilic Pneumonia Induced by Daptomycin. J. Infect. 2007, 54, e211-e213. [CrossRef]

11. Miller, B.A.; Gray, A.; Leblanc, T.W.; Sexton, D.J.; Martin, A.R.; Slama, T.G. Acute Eosinophilic Pneumonia Secondary to Daptomycin: A Report of Three Cases. Clin. Infect. Dis. 2010, 50, e63-e68. [CrossRef] [PubMed]

12. Kakish, E.; Wiesner, A.M.; Winstead, P.S.; Bensadoun, E.S. Acute Respiratory Failure Due to Daptomycin Induced Eosinophilic Pneumonia. Respir. Med. CME 2008, 1, 235-237. [CrossRef]

13. FDA Drug Safety Communication: Eosinophilic Pneumonia Associated With the Use of Cubicin (Daptomycin). Available online: https:/ / www.fda.gov/drugs/postmarket-drug-safety-information-patients-and-providers/fda-drug-safety-communicationeosinophilic-pneumonia-associated-use-cubicin-daptomycin (accessed on 6 December 2021).

14. Durante-Mangoni, E.; Andini, R.; Parrella, A.; Mattucci, I.; Cavezza, G.; Senese, A.; Trojaniello, C.; Caprioli, R.; Diana, M.V.; Utili, R. Safety of Treatment With High-Dose Daptomycin in 102 Patients With Infective Endocarditis. Int. J. Antimicrob. Agents 2016, 48, 61-68. [CrossRef] [PubMed]

15. Estes, K.S.; Derendorf, H. Comparison of the Pharmacokinetic Properties of Vancomycin, Linezolid, Tigecyclin, and Daptomycin Eur. J. Med. Res. 2010, 15, 533-543. [CrossRef] [PubMed]

16. Bennett, W.M.; Aronoff, G.R.; Morrison, G.; Golper, T.A.; Pulliam, J.; Wolfson, M.; Singer, I. Drug Prescribing in Renal Failure: Dosing Guidelines for Adults and Children, 5th ed.; American College of Physicians: Philadelphia, PA, USA, 1983.

17. Kullar, R.; McClellan, I.; Geriak, M.; Sakoulas, G. Efficacy and Safety of Daptomycin in Patients with Renal Impairment: A Multicenter Retrospective Analysis. Pharmacotherapy 2014, 34, 582-589. [CrossRef] [PubMed] 